\title{
A-Cross: An Accessible Crossword Puzzle for Visually Impaired Users
}

\author{
Stavroula Ntoa ${ }^{1}$, Ilia Adami ${ }^{1}$, Giannis Prokopiou ${ }^{2}$, Margherita Antona ${ }^{1}$, \\ and Constantine Stephanidis ${ }^{1,2}$ \\ ${ }^{1}$ Foundation for Research and Technology - Hellas (FORTH) \\ Institute of Computer Science \\ N. Plastira 100, Vassilika Vouton, GR-700 13 Heraklion, Crete, Greece \\ ${ }^{2}$ University of Crete, Department of Computer Science, Greece \\ \{stant, iadami, antona, cs\} @ics. forth.gr
}

\begin{abstract}
Crossword puzzles are a very popular word game, with high recreational and educational value, which can also be used as mental work-out tools, assisting in the prevention of age-related diseases, such as Alzheimer's disease. Despite their high value and although users with visual impairments constitute an important market share, there are only few accessible crossword puzzles. Even these, however, are limited in providing alternative input and output modalities for users with disabilities and do not support equitable use, simplicity and intuitiveness, especially for blind users. This paper presents A-Cross, an accessible crossword puzzle for visually impaired users, aiming to support word solving in a novel and usable way. The evaluation process that was followed in order to assess the usability and accessibility of a functional yet early prototype of the A-Cross puzzle is also described.
\end{abstract}

Keywords: Accessible, crossword, visually impaired, blind, puzzle, game, evaluation.

\section{Introduction}

Crossword puzzles are a very popular word game, dated since 1913 when they first appeared in the Sunday New York World. Ever since they have spread worldwide, to the point that almost every newspaper nowadays includes a crossword puzzle, while several dedicated books and monthly magazines are being published and purchased by an enthusiastic audience. Modern versions of crosswords include computer games, which beside their recreational character, may also act as educational tools and as mental work-outs.

Crossword puzzles have been employed for educational purposes since the $1920 \mathrm{~s}$ [11]. Childers [3] reports the use of crosswords in an introductory sociology course as an exercise that would help students identify those areas in which they needed additional work, and as a device to help them study for the final exam. Childers also discusses the evaluation that was carried out with 99 students, regarding the use of crosswords. According to the results, $96 \%$ of the evaluation participants found 
crosswords helpful, $47 \%$ reported their usefulness in studying for the final exam and $26 \%$ stated that crosswords helped them identify areas for further study or clarified certain concepts. Franklin et. al [5] used games and puzzles as a tool for stimulating class discussion of study topics and concluded that crossword puzzles are useful aids to learning. Weisskireh [16] used crossword puzzles as exam review tools and concluded that an appropriately designed crossword puzzle can provide an easy and engaging way for students to review concepts when preparing for a test. Wise [18] discusses the use of crossword puzzles as a tool for students' self-assessment, and reports positive student attributes and their unanimous confidence that crosswords could be employed by teachers to support learning.

On the other hand, the use of crosswords as memory aids is also widely recognised and an important corpus of research has been devoted to this area. For example, a study that was carried out with 801 participants between 1994 and 2001 analysed participants' cognitive activity and Alzheimer's Disease (AD) incidents and reported that frequent participation in cognitively stimulating activities (which include crossword solving) is associated with reduced risk of AD [17]. Another study [14] carried out with 469 participants between 1980 and 2001, reported that elderly persons who did crossword puzzles four days a week had a risk of dementia that was 47 percent lower than that among subjects who did puzzles once a week.

Given the high value of crosswords as recreational, education and mental work-out tools, their accessibility is of high importance for people with disabilities, who constitute an important market share [4, 15, 19]. Crossword accessibility is an interesting issue for blind users, who need alternative access to the game content, but also to understand the relations between words and to receive appropriate feedback regarding the input that they have provided and in general about the results of their actions. Although there are some crossword puzzles aiming to be accessible for blind users, their main problem is that they only provide alternative output through audio and access through keyboard, instead of also facilitating users in understanding how words are interrelated in the crossword and assisting them to create a representative mental model of the crossword.

This paper presents A-Cross, an accessible crossword puzzle for visually impaired users, aiming to support word solving in a novel and usable way. The next sections provide a discussion of accessible crossword puzzles for blind users, present the A-Cross puzzle and describe the evaluation process that was followed in order to assess the usability and accessibility of a functional early prototype of the puzzle. The last section of the paper discusses the conclusions from the conducted work and highlights future plans.

\section{Accessible Crossword Puzzles for Blind Users}

Several crossword puzzles are available online on the web or as standalone applications, few of which however are accessible to blind users. The main accessibility needs of blind users typically include audio output, as well as keyboard access to the application functionality, but the mere application of these techniques is not adequate to constitute a crossword puzzle genuinely accessible to blind users. In order to 
illustrate the above, three accessible crossword puzzles are further studied and their main accessibility features are presented in detail.

Talking Word Puzzles [1] is a puzzle application aimed at blind and visually impaired computer users. Output to blind users is inherently provided through speech, without the need for specialised software, mainly by announcing the clue that the user has selected to solve, as well as the letters typed by the user when solving a clue. Furthermore, when a clue is announced, the number of letters composing the correct solution is also declared. Navigation is supported through keyboard, namely with the Tab key, which moves the focus to the next clue, and the Enter key, which moves the focus from a clue to the corresponding crossword field, allowing the user to type the solution. It should be noted that the standard crossword format is retained, and therefore clues are organized in two dimensions, across and down, while each clue is identified by a number. Alternatively, users may navigate in the crossword grid, using the arrow keys, while the application announces each letter the cursor crosses. If there is a number in the square to which the cursor moves, indicating the beginning of a new word, the program announces that number, too. At that time, the user can directly move the focus from the grid to corresponding clue, by pressing the Enter key. Finally, the user may retrieve information regarding the number of remaining words in order to solve the puzzle.

Tackle Reading Crossword Puzzle [6] was developed for elementary school children, providing keyboard access and screen reader support for blind students. Users can access the clues' list sequentially through the Tab key, while at any time they may easily access a clue related to the active one, using the arrow keys. Two clues are related if their answers are crossing each other on the crossword grid.

Finally, the Blind Gamers (BG) Crossword puzzle [2] provides keyboard support and speech output to users without the need for a screen reader. Players are first presented with the "across" clues list and can change to the "down" clues' list by selecting an appropriate keyboard shortcut. At any time during the game play, players may switch to the related clues intersecting the currently active word, through an appropriate keyboard combination. Overall, the game provides to players a large number of options aiming to enhance its accessibility and usability; however, this is achieved through a large number of keyboard shortcuts, which may cause frustration to beginner game users. Another important drawback that has been noted is that if there are already filled-in letters for the word that currently receives focus (e.g., if some of its intersecting clues have been solved) the existing word data are not announced to the player and as a result the solving process may advance more slowly.

Careful reviewing of the aforementioned accessible crossword puzzles indicated that, although it is possible for the target user group to interact with the game, there are several concerns that should be taken into account, in order for the game to be actually usable. A-Cross was designed with these issues in mind, aiming to be both accessible and highly usable by blind users.

\section{The Design and Development of A-Cross}

A-cross was designed with the aim to cater for blind users' needs, having in mind not only to provide an accessible interface, but also to adhere to the "Design for All" 
principles [13]. Particular attention was therefore paid in order to create a crossword that would ensure equitable use, simplicity and intuitiveness for the specified target group.

In this context, the crosswords concept as well as issues regarding their representation on a grid were revisited and assessed in relation to the blind users' needs. In more detail, the simple spatial representation of words in the two dimensions, which is a concept tightly related to crosswords, turned out to be ineffective for blind users. As a result, a challenge during the design of A-cross was to provide blind users with information equivalent to all the visual hints conveyed to sighted users by the actual crossing of words on the grid. Furthermore, additional cues obtained by sighted users, such as which letters have been already filled in for a word, or the number of letters constituting a word, should also be effectively communicated to blind users of A-cross, allowing them to develop an efficient strategy for crossword solving.

In order to address the aforementioned issues, A-cross does not only provide clues classified in two dimensions (across / down), clues are instead presented according to their relevance to the currently solved clue. The term relevance is used here to denote all the words that actually cross the current word the user is solving in a typical crossword grid. Therefore, an algorithm for the automatic suggestion of the next clue to be announced is applied, allowing blind users to efficiently solve the puzzle. As illustrated in the algorithm flowchart in Fig. 1, if the current clue has been solved, then the next related unvisited clue with the fewest missing letters is automatically suggested. Unvisited clues are considered those which the user has never listened to before. If the user wishes to move to the next clue without solving the currently suggested one, then the same decision making process is followed. When there are no related clues available, then the next sequential clue in the clue list is suggested.

Additional features of A-cross aiming to improve its usability and accessibility include:

- Automatic announcement of the number of letters constituting the corresponding answer, when a clue is announced

- Automatic announcement of the letters that have been already completed for a clue, by solving other related clues

- Audio feedback for the correct answer to a clue

- Audio feedback for correctly typed letters when answering a clue. This feature can be activated or not, according to each individual user's preferences

- Elimination of the already solved clues from the clues list. This is an important feature, aiming to speed up the crossword solving process, by discarding all the unnecessary information. The reasoning behind this feature is to simulate the mental model of a sighted user, who would not revisit a correctly answered clue. This feature is initially automatically enabled, however users may disable it.

- Enlarged interface for low vision users and high color contrast interface for color blind users.

It should be mentioned that A-cross was designed and implemented as a dual user interface [9], allowing access to both sighted and blind users at the same time. Currently, a fully interactive prototype of the game has been implemented, in order to allow its evaluation. The implementation was carried out in C\# with Microsoft Visual 
Studio 2010, while the text to speech facilities required for the narrator are available through the Microsoft Speech API 5.3 ${ }^{1}$. Keyboard navigation is supported for blind users through the $\mathrm{TAB}$ and arrow keys (for lists).

Once the game is installed, the default settings have the narrator enabled, ensuring first-time access for blind users. Players may disable the narrator and adjust the visual layout according to their preferences when creating or editing their player profile (Fig. 2a). Additional profile options include high color contrast and interface enlargement, in order to make the game accessible for color-blind and low vision users. Furthermore, every time the game is initiated, it considers as active the last

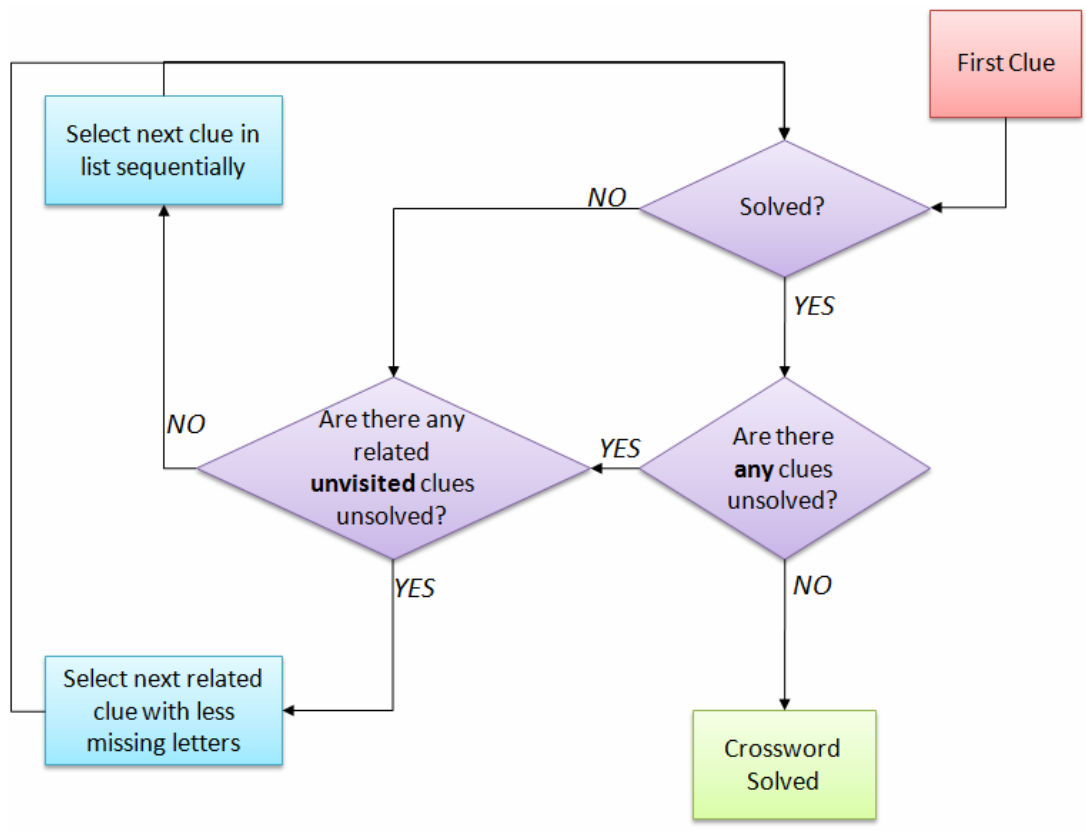

Fig. 1. Flowchart presenting the algorithm for the automatic suggestion of the next clue to be solved
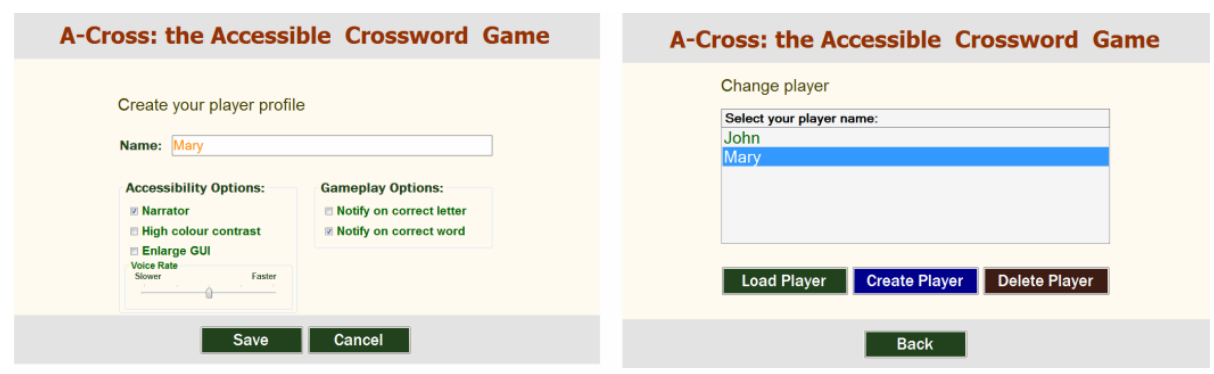

Fig. 2. (a) Creation of new player profile (b) Player selection

\footnotetext{
${ }^{1}$ http://msdn.microsoft.com/en-us/library/ms723627\%28v=VS.85\%29.aspx
} 


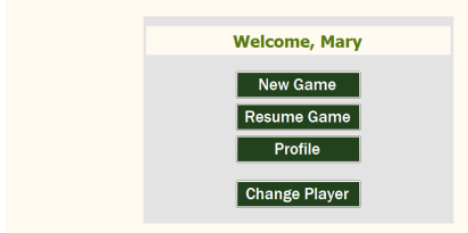

Scores

Instructions

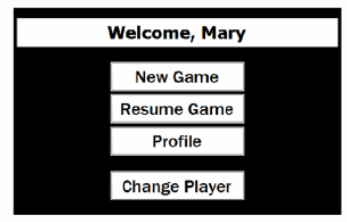

Fig. 3. Main menu (a) standard (b) high color contrast

player's profile. Users are however allowed to change (Fig. 2b) the current player profile through an option in the main menu (Fig. 3).

Players are able to select the game they wish to play through a list of available crosswords. In the main game screen (Fig. 4) a horizontal menu allows users to pause the game, solve the game, get letter or word hint, change their profile settings (e.g., enable / disable the narrator), change volume settings and return to main menu.
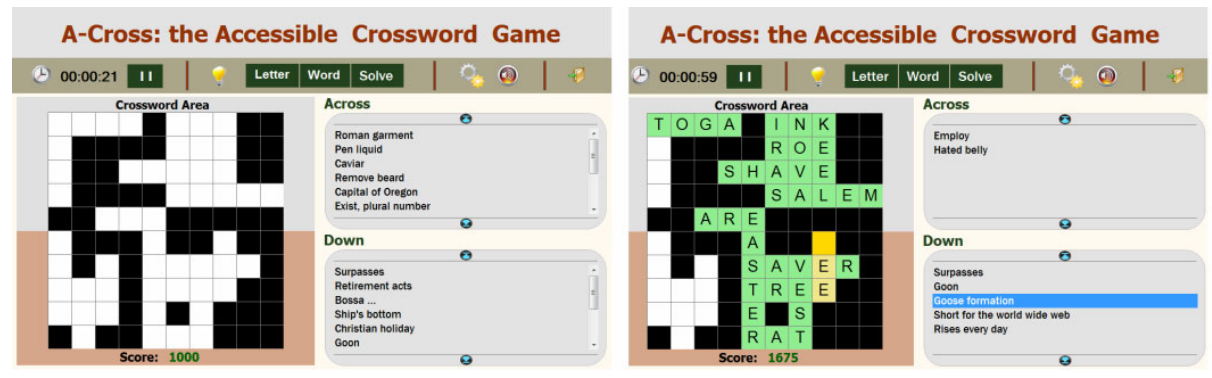

Fig. 4. Crossword screen (a) no clues solved (b) the word clue lists get smaller as the user solves the puzzle

The A-cross prototype is deployed with four crosswords embedded. However, an important feature of A-cross is that the supported crosswords are not hard-coded; instead, they are available through external .xml files, allowing thus updating A-cross with an infinite number of new crosswords.

\section{Usability and Accessibility Evaluation of the A-Cross game}

Once the first working crossword puzzle prototype was developed, an expert based evaluation was conducted in order to test the game application against usability and accessibility standards. Expert and cognitive walkthroughs are two of the methods of expert based evaluation which involve having one or more accessibility/usability experts interact with the application looking for accessibility and usability problems and areas that may potentially cause confusion and trouble to the user [10]. Expert based evaluations are very valuable for identifying most usability and accessibility 
issues before conducting real user based evaluations. They also serve as a good alternative when it is difficult to find actual or potential users for evaluation [12]. The A-cross crossword puzzle was designed primarily for use by users with blindness, partial blindness, and other vision impairments. Considering the fact that finding local users who are blind, have some computer experience, and speak English is not an easy task, when such testers are found, it is imperative to make the most out of their testing experience and not waste their time with obvious inefficiencies that could have been caught by an accessibility expert. Thus, at this preliminary stage of the application life cycle, expert based evaluation was chosen as the method of testing and user based testing with blind users was reserved for a later stage after the first improvements and adjustments are made on the game.

\subsection{Expert / Cognitive Walkthrough}

The evaluation was conducted by three usability and accessibility experts. Each one of them carried out a thorough inspection of the game functionality and overall design and wrote their findings in a report. The discovered problems were then aggregated on a single report, and each item was rated individually by each expert using a scoring scale from 0 (not a usability problem) to 4 (usability catastrophe) [8]. An average score was given for each item on the list. The most severe issues found by the experts are discussed in more details below.

Expert Evaluation Results. The evaluators found a total of thirteen issues that a blind user might encounter. Of those thirteen issues, three of them were rated pretty high (received an average score of greater than 3.5) on the scoring scale and their resolution was thus deemed of critical importance. These issues are described below:

- Issue 1. Severity score= 4: There are no clear instructions for the blind user on how to proceed with solving the puzzle once it is loaded. In particular, when the puzzle gets loaded the narrator says "The game started. This crossword puzzle has 21 word clues that you need to solve. Happy Gaming". Since proceeding to the crossword area and interacting with the clues is carried out with the arrow keys and not the TAB key which is reserved for the menu options (pause, hints, settings), the blind users may be confused and not able to proceed. The experts recommended that the problem would be easily solved by including brief instructions on how to proceed to the game after the initial message.

- Issue 2. Severity score=3.83: Another point that received very high marks by all experts is that there is no easy or obvious way for the user to have the word clue repeated to them on command after it is read by the narrator the first time. Considering the fact that the spoken output by the narrator is not always clear, such a function becomes imperative. The experts noted that the omission of this functionality will frustrate the blind users who want to be able to go back and forth in a sentence if they don't understand it the first time. A shortcut key $(\mathrm{Ctrl}+\mathrm{R})$ that would repeat the last word clue was suggested as a remedy.

- Issue 3. Severity score= 3.63. As shown in Fig. 4, A-Cross provides the users with two help options, namely to reveal a letter and to reveal a word. Although the hint is provided to the user, the system does not provide appropriate feedback regarding the letter or the word that was solved, creating confusion to blind users. Besides, 
announcing the requested letter or word after it is filled out by the system, experts also suggested not having the system automatically move to the next word clue, but allow the user to do so at his/her own time.

- Issue 4. Severity score $=3.16$. Experts noted that although the A-Cross layout resembles a regular crossword, there are no numbers marked either on the crossword area, or next to the word clues.

Other suggestions included minor graphical user interface improvements, the addition of keyboard shortcuts for instant access to game options during crossword solving (e.g., voice volume, speed of speech) and elimination of a few functional errors that occurred.

Features of A-Cross that were positively commented by the experts include the simplicity of the graphical interface, the simplicity of keyboard alternatives, as well as the expected learnability and ease of use even by beginners (once the identified problems will be eliminated).

Finally, one question raised by the experts was if the A-Cross model matches the mental model of players, since for example the next word to be solved is automatically suggested by the game or since once a word clue is solved correctly it disappears from the clues' list. However, the mental model of blind players could not be adequately estimated by the experts and a user testing was deemed necessary in this respect. An additional issue that user testing is expected to reveal involves the effectiveness and efficiency of the narrator, in order to provide appropriate guidance and feedback to blind players.

As already mentioned, plans for user-based testing on A-cross are in the near future and after the first round of improvements on the design of the game has finished, given that usability testing is the most fundamental evaluation method, because it provides direct input on how the user perceives and interacts with the application or interface that is being tested [7]. However, one user based test with a blind user that fit perfectly the profile of a game user was conducted after the expert evaluation was completed. Even though testing with one user is not enough to draw any final conclusions, it does help in confirming the experts' findings. The findings of that test are discussed in the next section.

\subsection{Preliminary User Testing with Blind User}

The user was a female in the age range of 25-30, very familiar with using assistive technologies on a computer and fluent in English. The evaluation was carried out in a usability evaluation lab and the session was recorded by a camera, after a signed permission was acquired by the participant.

Initially the participant was introduced to the purpose of the evaluation and was instructed to think aloud during each task, letting the evaluator know what she was thinking prior, during, and after each action. The experimenter asked the evaluator to carry out a number of tasks, which included the following: (1) Start the application and create a new user profile; (2) Select the puzzle "Easy" and load the puzzle; (3) Solve as many words as possible. When coming across a word clue that she did not know how to solve, the user was instructed to find a way to get a hint from the system; (4) Exit the application, restart it and continue from where it had been left off. 
During the test the experimenter was observing and taking notes on what was happening. Once the process was completed, the user was debriefed and thanked for her valuable participation.

The results verified the findings of the expert-based evaluation, namely that the narrator did not provide adequate feedback when a word was solved and it was unclear when it moved to a new clue, and that repeating the requested word clue at user's will was essential. Furthermore, she noted that it mattered to a blind user to know if a clue was horizontal or vertical, otherwise there is no sense in having two lists and that they could have been in one list.

It should be noted, however, that a single user based evaluation is definitely not enough to draw conclusions, but it certainly affirms the need for more testing and confirms the experts' findings.

\section{Conclusions and Future Work}

This paper has presented A-Cross, an accessible crossword game for blind and visually impaired users. Aiming to enhance its usability and accessibility by blind users, the game automatically suggests the next clue to be announced to the blind player, by selecting the next clue related to the current word with the fewest missing letters. A fully interactive prototype of A-Cross has been implemented as a dual interface, and it has been evaluated by usability and accessibility experts and by one blind user.

The usability evaluation pointed out a number of usability and accessibility errors that should be corrected before proceeding to a user-based evaluation, which is among the future work plans. User testing is considered irreplaceable in order to successfully assess whether A-Cross meets its goals, and whether the proposed automatic clue suggestion algorithm addresses blind users' needs and ensures an efficient and pleasant gameplay.

\section{References}

1. American Printing House for the Blind, Talking Word Puzzles, http://www.aph.org/tech/pz_info.htm (retrieved February 6, 2011)

2. Blindgamers. BG Crossword Puzzle, http: / / www . omninet. net. au/ irhumph/blindgamers . htm\#CROSSWORD SAPI (retrieved February 7, 2011)

3. Childers, C.D.: Using Crossword Puzzles as an Aid to Studying Sociological Concepts. Teaching Sociology 24(3), 231-235 (1996)

4. European Commission: Report of the Inclusive Communications (INCOM) subgroup of the Communications Committee (COCOM). COCOM04-08 (2004)

5. Franklin, S., Peat, M., Lewis, A.: Nontraditional interventions to stimulate discussion: The use of games and puzzles. Journal of Biological Education 37(2), 79-84 (2003)

6. Jordan, M.: Strategies for Building Accessible Learning Web Applications and Games with Macromedia Flash MX. In: CSUN 2005 Technology \& Persons with Disabilities Conference (2005),

http: / / www . csun. edu/cod/conf / $2005 /$ proceedings / 2330 . htm (retrieved February 6, 2011) 
7. Nielsen, J.: Mental Models. In Jakob Nielsen's Alertbox 2010 article, http://www. useit.com/alertbox/mental-models.html (retrieved February 8,2011$)$

8. Nielsen, J.: Usability Engineering. Morgan Kaufmann, San Francisco (1993)

9. Savidis, A., Stephanidis, C.: Developing dual user interfaces for integrating blind and sighted users: the HOMER UIMS. In: Proceedings of the ACM Conference on Human Factors in Computing Systems (CHI 1995), pp. 106-113. ACM Press, New York (1995)

10. Petrie, H., Bevan, N.: The Evaluation of Accessibility, Usability, and User Experience. In: Stephanidis, C. (ed.) The Universal Access Handbook, pp. 10-20. CRP Press Taylor and Francis Group, Abington (2009)

11. Schafer, J.C., Behymer, J.: Cross Purposes: Computer-Generated Crossword Puzzles Link Popular Pastime with Technical Learning. Vocational Education Journal 67(5), 36-37 (1992)

12. Schroeder, W.: Scheduling Hard-to-Find Users. User Interface Enginnering (1998), http://www.uie.com/articles/scheduling_users (retrieved February 8, 2011)

13. The Center for Universal Design: The Principles of Universal Design, Version 2.0. Raleigh, NC: North Carolina State University (1997)

14. Verghese, J., Lipton, B.R., Katz, J.M., Hall, B.C., Derby, A.C., Kuslansky, G., Ambrose, F.A., Sliwinski, M., Buschke, H.: Leisure Activities and the Risk of Dementia in the Elderly. The New England Journal of Medicine 348(25), 2508-2516 (2003)

15. USCENSUSBUREAU. Americans with disabilities: 2002 (2006), http: / /www. census.gov/prod/2006pubs /p70-107.pdf (retrieved February 7, 2011)

16. Weisskireh, R.S.: An analysis of instructorcreated crossword puzzles for student review. College Teaching 54(1), 198-201 (2006)

17. Wilson, R., Mendes de Leon, S., Barnes, C.F., Schneider, L.L., Bienias, A.J., Evans, L.J., Bennett, A.D., Participation, A.D.: in Cognitively Stimulating Activities and Risk of Incident Alzheimer Disease. Journal of the American Medical Association 287(6), 742-748 (2002)

18. Wise, A.: Web-based crossword puzzles support revision. Active Learning in Higher Education 2(2), 180-188 (2001)

19. World Health Organization: Fact Sheet No282: Visual Impairments and blindness (2009), http: //www. who. int/mediacentre/factsheets/fs282/en/index.htm 1 (retrieved February 7, 2011) 